\title{
Mutant small heat-shock protein 27 causes axonal Charcot-Marie-Tooth disease and distal hereditary motor neuropathy
}

\author{
Oleg V Evgrafov ${ }^{1}$, Irena Mersiyanova ${ }^{2}$, Joy Irobi ${ }^{3}$, Ludo Van Den Bosch ${ }^{4}$, Ines Dierick ${ }^{3}$, Conrad L Leung ${ }^{5}$, \\ Olga Schagina ${ }^{2}$, Nathalie Verpoorten ${ }^{3}$, Katrien Van Impe ${ }^{6}$, Valeriy Fedotov ${ }^{7}$, Elena Dadali ${ }^{2}$, \\ Michaela Auer-Grumbach ${ }^{8}$, Christian Windpassinger $^{8}$, Klaus Wagner $^{8}$, Zoran Mitrovic ${ }^{9}$, David Hilton-Jones ${ }^{10}$, \\ Kevin Talbot ${ }^{11}$, Jean-Jacques Martin ${ }^{12}$, Natalia Vasserman ${ }^{2}$, Svetlana Tverskaya ${ }^{2}$, Alexander Polyakov ${ }^{2}$, \\ Ronald K H Liem ${ }^{5}$, Jan Gettemans ${ }^{6}$, Wim Robberecht ${ }^{4}$, Peter De Jonghe ${ }^{3,13}$ \& Vincent Timmerman ${ }^{3}$
}

\begin{abstract}
Charcot-Marie-Tooth disease (CMT) is the most common inherited neuromuscular disease and is characterized by considerable clinical and genetic heterogeneity ${ }^{1}$. We previously reported a Russian family with autosomal dominant axonal CMT and assigned the locus underlying the disease (CMT2F; OMIM 606595) to chromosome 7q11-q21 (ref. 2). Here we report a missense mutation in the gene encoding $27-\mathrm{kDa}$ small heat-shock protein B1 (HSPB1, also called HSP27) that segregates in the family with CMT2F. Screening for mutations in HSPB1 in 301 individuals with CMT and 115 individuals with distal hereditary motor neuropathies (distal HMNs) confirmed the previously observed mutation and identified four additional missense mutations. We observed the additional HSPB1 mutations in four families with distal $\mathrm{HMN}$ and in one individual with CMT neuropathy. Four mutations are located in the Hsp20- $\alpha$-crystallin domain, and one mutation is in the $C$ terminal part of the HSP27 protein. Neuronal cells transfected with mutated $H S P B 1$ were less viable than cells expressing the wild-type protein. Cotransfection of neurofilament light chain (NEFL) and mutant HSPB1 resulted in altered neurofilament assembly in cells devoid of cytoplasmic intermediate filaments.
\end{abstract}

CMT belongs to the hereditary motor and sensory neuropathies and is characterized by degeneration of peripheral nerves. CMT is further subdivided into CMT1 and CMT2, the demyelinating and axonal forms, respectively ${ }^{1}$. CMT2 is genetically heterogeneous, and eight loci

and five genes have been reported to cause dominantly inherited CMT2 (refs. 2-10). Affected individuals in the Russian family have progressive symmetrical weakness and atrophy of distal limb muscles, initially involving the legs and particularly the peroneal muscles. They have depressed or absent tendon reflexes and mild to moderate distal sensory abnormalities. The onset age is $15-25$ years $^{2}$. We carried out a haplotype analysis of this family using short tandem repeat markers and narrowed the locus underlying the disease to $10 \mathrm{cM}$ between markers D7S672 and D7S806 (data not shown). From this region we selected five candidate genes, which encode huntingtin-interacting protein (HIP1), atrophin-1 interacting protein 1 (AIP1), cytoplasmic linker 2 (CYLN2), protein tyrosine phosphatase, nonreceptor type 12 (PTPN12) and small heat-shock 27-kDa protein B1 (HSPB1). We sequenced all known exons and intron-exon boundaries of each gene

Table 1 Mutations in HSPB1 in families with CMT2 or distal HMN

\begin{tabular}{lcccc}
\hline Family & Diagnosis & Origin & $\begin{array}{c}\text { Nucleotide } \\
\text { change }\end{array}$ & $\begin{array}{c}\text { Amino acid } \\
\text { change }\end{array}$ \\
\hline PN-474.1 & Distal HMN & Belgium & $379 \mathrm{C} \rightarrow \mathrm{T}$ & $\mathrm{R} 127 \mathrm{~W}$ \\
CMT2F & CMT2 & Russia & $404 \mathrm{C} \rightarrow \mathrm{T}$ & $\mathrm{S} 135 \mathrm{~F}$ \\
CMT-150 & Distal HMN & UK & $404 \mathrm{C} \rightarrow \mathrm{T}$ & $\mathrm{S} 135 \mathrm{~F}$ \\
PN-269.1 & CMT2 & Belgium & $406 \mathrm{C} \rightarrow \mathrm{T}$ & $\mathrm{R} 136 \mathrm{~W}$ \\
CMT-263 & Distal HMN & Croatia & $452 \mathrm{C} \rightarrow \mathrm{T}$ & $\mathrm{T} 151 \mathrm{I}$ \\
CMT-391 & Distal HMN & Austria & 545C $\rightarrow \mathrm{T}$ & $\mathrm{P} 182 \mathrm{~L}$ \\
\hline
\end{tabular}

${ }^{1}$ Department of Psychiatry, New York State Psychiatric Institute/Research Foundation for Mental Hygiene, Unit 28, 1051 Riverside Drive, New York, New York 10032 , USA. ${ }^{2}$ DNA-Diagnostics Laboratory, Research Center For Medical Genetics, Moscow, Russia. ${ }^{3}$ Department of Molecular Genetics, Flanders Interuniversity Institute for Biotechnology, University of Antwerp, Antwerpen, Belgium. ${ }^{4}$ Laboratory for Neurobiology, Department of Experimental Neurology, University of Leuven, Leuven, Belgium. ${ }^{5}$ Department of Pathology, Columbia University, College of Physicians and Surgeons, New York, USA. ${ }^{6}$ Department of Medical Protein Research, Flanders Interuniversity Institute for Biotechnology, Ghent University, Ghent, Belgium. ${ }^{7}$ Genetic Counseling Department, Diagnostic Center, Voronezh, Russia. ${ }^{8}$ Institute of Medical Biology and Human Genetics, Medical University Graz, Austria. ${ }^{9}$ Centre for Neuromuscular Diseases, Department of Neurology, Clinical Hospital Centre Zagreb, University School of Medicine, Zagreb, Croatia. ${ }^{10}$ Department of Clinical Neurology, Radcliffe Infirmary, Oxford, UK. ${ }^{11}$ Department of Human Anatomy and Genetics, University of Oxford, UK. ${ }^{12}$ Born-Bunge Foundation, University of Antwerp, Antwerpen, Belgium. ${ }^{13}$ Division of Neurology, University Hospital Antwerpen, Antwerpen, Belgium. Correspondence should be addressed to O.V.E. (Evgrafo@pi.cpmc.columbia.edu).

Published online 2 May 2004; doi:10.1038/ng1354 
and did not find any disease-associated mutations in $H I P 1, A I P 1$, CYLN2 or PTPN12. But in exon 2 of HSPB1 we found a $404 \mathrm{C} \rightarrow \mathrm{T}$ heterozygous transition leading to a S135F missense mutation in HSP27. This mutation segregated perfectly with the CMT phenotype.

We next screened an additional 301 unrelated individuals with CMT. Because mutations of HSP22 (also called HSPB8, an interacting partner of HSP27; ref. 11) were identified in individuals with distal HMN type II (OMIM 158590; ref. 12), we also screened 115 unrelated individuals with distal HMN. Distal HMN is a pure motor peripheral neuropathy that closely resembles CMT2, apart from the absence of sensory abnormalities in distal $\mathrm{HMN}^{13,14}$. We found the same $404 \mathrm{C} \rightarrow \mathrm{T}$ mutation in four Russian families (Voronezh province and Moscow) with CMT2 and in one English family with distal HMN. Comparison of the disease-segregating haplotypes using five short tandem repeat markers (D7S672, D7S2490, D7S2470, D7S2455 and D7S675) flanking HSPB1 indicated that the four Russian families probably had a common ancestor but that the English family was not closely related to them (data not shown). In addition, we found four other missense mutations in HSPB1 in families with CMT2 or distal HMN (Table 1 and Fig. 1a). These muta- tions segregated perfectly with the axonal CMT and distal HMN phenotypes in all families and were absent in 200 control individuals of European descent.

Four of the five missense mutations of HSP27 (R127W, S135F, R136W and T151I) occur in the Hsp20- $\alpha$-crystallin domain, which is highly conserved among the human small heat-shock proteins ${ }^{15}$ (Fig. 1b). The mutant amino acid residues Arg127, Ser135 and Arg136 are located close to residue Arg140. This residue corresponds to Arg120 in $\alpha \mathrm{B}$-crystallin (also called CRYAB and HSPB5), which is mutant in desmin-related myopathy (OMIM 601419; ref. 16), and Arg116 in $\alpha$ Acrystallin (also called CRYAA or HSPB4), which is mutant in congenital cataract (OMIM 123580; ref. 17). The Arg116 residue is crucial for the structural and functional integrity of the $\alpha \mathrm{A}$-crystallin ${ }^{18}$. In addition, mutation of residue Cys 137 in the Hsp20- $\alpha$-crystallin domain of HSP27 abrogates its binding to cytochrome c, resulting in caspase activation and apoptosis ${ }^{19}$. The fifth HSP27 missense mutation (P182L) occurs in the variable C-terminal tail of the $\operatorname{protein}^{15}$ (Fig. 1b). The Pro182 residue is close to two dominant negative mutations in the Cterminal region of $\alpha \mathrm{B}$-crystallin that cause myofibrillar myopathy and affect the solubilization and chaperone function of the molecule 20,21 . All five mutations occur in amino acids that are conserved in HSPB1 orthologs (Fig. 1c). Northern-blot analysis confirmed that HSPB1 was ubiquitously expressed in human tissues (Supplementary Fig. 1 online). In addition, RT-PCR analysis of mRNA obtained from dorsal root ganglia and ventral horn of mouse embryos ( $13 \mathrm{~d}$ old) showed $H s p b 1$ expression in sensory ganglia and the ventral horn (Supplementary Fig. 1 online).

Because hereditary peripheral neuropathies are caused by premature axonal loss, possibly due to neuronal degeneration, we investigated the effect of the HSP27 S135F mutation on cell survival. We transiently transfected mouse neuroblastoma cells (N2a) with constructs expressing enhanced green fluorescent protein (EGFP)tagged wild-type or S135F mutant HSP27 and measured cell survival after $3 \mathrm{~h}, 24 \mathrm{~h}$ and $48 \mathrm{~h}$ using the MTS assay. This assay reflects cell viability and proliferation as well as integrity of the mitochondria. After $48 \mathrm{~h}$, the number of cells transfected with mutated HSPB1 was significantly lower than the number of cells transfected with wild-type HSPB1 $(P=0.024$; Fig. 2a). This difference was not due to varying expression levels of HSPB1 (Fig. 2b). In addition, transfection with mutated HSPB1 almost doubled the number of multinucleated $\mathrm{N} 2 \mathrm{a}$ cells compared with transfection with wild-type HSPB1 (Supplementary Fig. 2 online). This effect also occurred in non-neuronal cells (COS and HEK293T) transfected with mutated HSPB1 (data not shown).

Mutations in NEFL, encoding the neurofilament light chain, cause another variant of axonal CMT (CMT2E, OMIM 607684; ref. 5), and alterations in the formation of a normal intermediate filament 
Figure 2 Overexpression of mutant $H S P B 1$ reduces cell viability. (a) N2a cell viability determined $24 \mathrm{~h}$ and $48 \mathrm{~h}$ after transfection without vector (column 1 , dark blue), with empty vector (column 2, red), with wild-type HSP27 (column 3, yellow) or with S135F mutant HSP27 (column 4, light blue). Values were normalized to the measurement obtained $3 \mathrm{~h}$ after transfection. Data shown are mean + s.e.m. for five independent experiments. (b) Western blot showing expression of wild-type HSP27 (lane 2) and S135F mutant HSP27 (lane 3 ) in N2a cells $3 \mathrm{~h}, 24 \mathrm{~h}$ and $48 \mathrm{~h}$ after transfection. Lane 1 shows results for cells transfected with the empty vector. Blots were stripped and stained for $\beta$-actin as a loading control. The relative expression of wild-type HSP27 versus mutant HSP27 was $0.98,0.96$ and 0.74 after $3 \mathrm{~h}, 24 \mathrm{~h}$ and $48 \mathrm{~h}$, respectively $(n=3)$ and was not statistically different. a

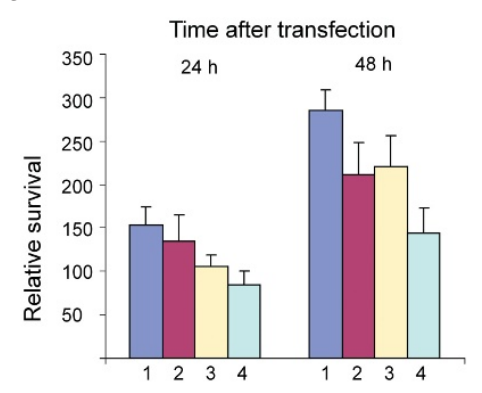

b

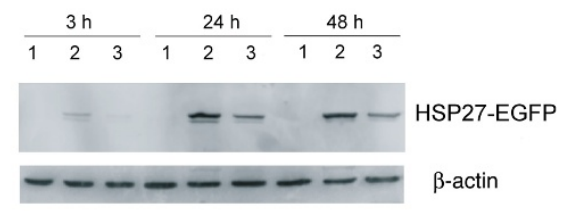

network caused by mutations in NEFL may contribute to the development of neuropathy ${ }^{22}$. We therefore studied whether mutant HSP27 affects neurofilament assembly. Human neurofilament light protein (NF-L) can self-assemble into homopolymeric filaments when expressed in SW13.cl.2 Vim ${ }^{-}$cells, an adrenal carcinoma cell line that does not contain an endogenous intermediate filament network ${ }^{22}$. We cotransfected human NEFL and wild-type HSPB1 in these cells and found that NF-L formed a thin filamentous network and, occasionally, thick filament bundles in the presence of overexpressed HSPB1. When we coexpressed NEFL with the S135F mutant HSPB1, however, transfected cells had an amorphous staining pattern consisting of NF-L aggregates and considerably less filamentous NF-L staining. We never observed this staining pattern in cells overexpressing wild-type HSPB1, suggesting that the S135F mutation in HSP27 affects neurofilament assembly (Fig. 3).

In summary, we found five distinct missense mutations in HSPB1 in six unrelated families with CMT2 or distal HMN. All mutations cosegregated perfectly with the disease phenotype in these families. The S135F mutation reduced viability of neuronal cells in vitro, suggesting that it is responsible for premature axonal degeneration, the direct cause of both CMT2 and HMN. The identification of mutations causing distal HMN type II (ref. 12) in HSP22, an interacting partner of HSP27, confirms the direct involvement of HSP27 in the etiology of these inherited peripheral neuropathies. Mouse Hspb1 was expressed in both ventral horn and sensory ganglia, and muta- tions in HSPB1 are associated with both distal HMN, a pure motor neuropathy, and CMT2F, a mixed sensory and motor neuropathy. This finding is not unexpected, as dominant mutations in GARS, encoding glycyl tRNA synthetase, are also associated with both types of neuropathies, CMT2D and distal HMN-V linked to chromosome 7 p14 (ref. 6).

Recent studies showed that heat-shock protein chaperones, including HSP27, have anti-apoptotic and cytoprotective properties and are often upregulated in several neurodegenerative diseases. HSP27 and $\alpha \mathrm{B}$-crystallin inhibit caspase activation ${ }^{19,23}$ and prevent aggresome formation by co-oligomerizing with mutant $\alpha \mathrm{B}$-crystallin ${ }^{16}$. Furthermore, upregulation of HSP27 is required for survival of injured sensory and motor neurons ${ }^{24}$ and also occurs in motor neurons of mice overexpressing mutant $\mathrm{Cu}, \mathrm{Zn}$ superoxide dismutase 1 (SOD1), a reliable model for human amyotrophic lateral sclerosis ${ }^{25-27}$. HSP27 is also a suppressor of polyglutaminemediated cell death ${ }^{28}$. In desmin-related myopathy, mutant $\alpha \mathrm{B}$-crystallin had a lower capacity to protect cells against heat shock and failed to serve as a chaperone in the assembly of desmin filaments ${ }^{20}$. Wild-type HSP27 is also involved in the organization of the neurofilament network ${ }^{29}$, which is important for maintaining the axonal cytoskeleton and transport. Here we showed that mutant HSP27 affects the neurofilament assembly. This adds CMT2F and distal HMN to the growing list of neuromuscular disorders, including peripheral neuropathies, in which dysfunction of the axon cytoskeleton and axonal transport seems to be the main part of
Figure 3 Assembly of NF-L in the presence of wildtype and mutant HSP27. SW13.cl.2Vim- cells were transiently transfected with $\mathrm{pCl}-\mathrm{hNFL}$ with pcDNA wild-type HSPB1 (a,b) or pcDNA S135F mutant HSPB1 (d,e). The transfected cells were stained with monoclonal antibody to NF-L NR4 $(\mathbf{a}, \mathbf{d})$ and polyclonal antibody to Hsp27 (b,e). The superimposed images with the NF-L staining (green) and Hsp27 (red) are shown in c and f. Scale bar, $20 \mu \mathrm{m}$. (g) The western blot of lysates obtained from transfected SW13.cl.2Vim- cells, probed with antibody to NF-L NR4 and polyclonal antibody to Hsp27, showed equal expression levels of wild-type and mutant HSP27. The blot was reprobed with antibody to $\beta$-tubulin as a loading control.
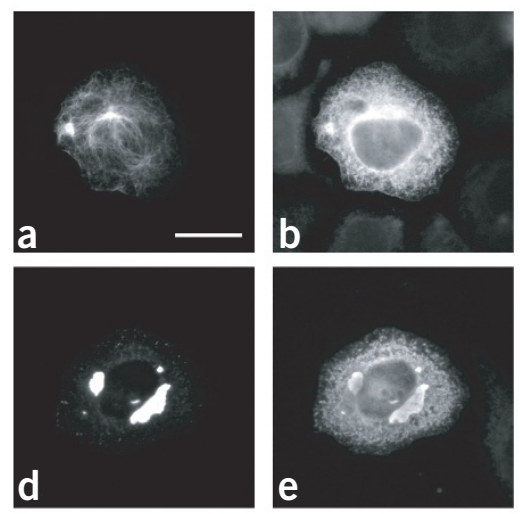
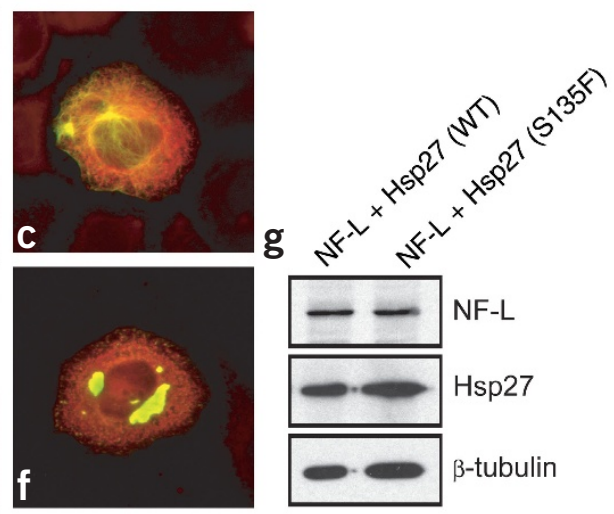
pathological mechanism ${ }^{1}$. The recent identification of HSPB8, encoding HSP22 which interacts with HSP27 (ref. 11), as another gene associated with distal $\mathrm{HMN}^{12}$ may help to identify the exact molecular process crucial for the disease, in which both proteins probably have an important role .

\section{METHODS}

Affected individuals. Our study included two families with CMT2 and four families with distal HMN (Supplementary Fig. 3 online). In the Russian family, we previously identified a locus on chromosome 7q11-q21 associated with $\mathrm{CMT}_{2} \mathrm{~F}^{2}$. The Belgian (PN-269, PN-474), English (CMT-150), Croatian (CMT-263) and Austrian (CMT-391) families were recently identified. From family members and control persons, we isolated genomic DNA from total blood samples using a standard extraction protocol. Informed consent was obtained from all family members, and the study was approved by the Institutional Review Board at the Universities of Antwerp, Graz, Moscow and Oxford.

Mutation analysis. We used the National Center for Biotechnology Information Entrez Genome Map Viewer, Ensembl Human Genome Server and GenBank database to find known genes, expressed-sequence tags and putative new genes in the CMT2F-linked region. We retrieved the exon-intron boundaries of the candidate sequences from the University of California Santa Cruz website or determined these by BLAST searches against the high-throughput genome sequences. We amplified all exons of HIP1, AIP1, CYLN2, PTPN12 and HSPB1 by PCR using intronic primers (sequences are available on request). We sequenced PCR products using either Big Dye Terminator kit on ABI373 sequencer (Applied Biosystems) or the DYEnamic ET Terminator Cycle Sequencing Kit (Amersham Pharmacia Biotech) on the ABI3700 sequencer (Applied Biosystems). We collected and analyzed data using the ABI DNA sequencing analysis software.

Expression analysis. We purchased a panel of normalized, first-strand cDNA preparations from poly $(\mathrm{A})^{+} \mathrm{RNA}$ from human tissues and cells from Clontech. We used gene-specific primers to construct a HSPB1 cDNA probe, which we used to hybridize the Human 12-lane Multiple Tissue Northern blot (Clontech). We also hybridized northern blots with an $A C T B$ cDNA probe (Clontech) as a control for RNA loading. We extracted total RNA from mouse muscle (Navy Medical Research Institute) using the Totally RNA Kit (Ambion) and carried out RT-PCR using the Random Primer DNA Labelling System (Life Technologies). We isolated ventral horns and dorsal root ganglia from 13-d-old mouse embryos. We extracted total RNA using the Totally RNA Kit (Ambion) and carried out RT-PCR using the SuperScript III First-Strand Synthesis System for RT-PCT (Invitrogen). Mouse Hsp b1 cDNA primers used to amplify a fragment of $634 \mathrm{bp}$ are available on request.

cDNA cloning and mutagenesis. We cloned the cDNA encoding full-length wild-type human HSPB1 (ref. 30) as a HindIII fragment into the pEGFP-C1 (Clontech) and pcDNA3.1V5/His TOPO (Invitrogen) vectors by PCR using the RZPD plasmid IRALp962H201 (HSPB1 cDNA) as templates. For site-directed mutagenesis of the HSPB1 mutation $404 \mathrm{C} \rightarrow \mathrm{T}$, we used the QuikChange sitedirected mutagenesis kit (Stratagene). The construction of pCI-hNFL has been previously described ${ }^{22}$. We verified constructs by direct DNA sequencing (Applied Biosystems)

Cell cultures and transfections. We cultured mouse neuroblastoma (N2a) cells (American Type Culture Collection, \#CCL131) in a 1:1 mix of Dulbecco's modified Eagle medium and F12 medium with glutamax (Gibco Invitrogen) supplemented with nonessential amino acids, penicillin $\left(100 \mathrm{U} \mathrm{ml}^{-1}\right)$, streptomycin $\left(100 \mu \mathrm{g} \mathrm{ml}^{-1}\right)$ and $10 \%$ fetal calf serum. Transfection was done using the Nucleofector technology (Amaxa). We collected exponentially growing cells and resuspended them at a concentration of $2 \times 10^{6}$ cells per $100 \mu \mathrm{l}$ in a cuvette containing Nucleofector solution T. We added plasmid DNA $(5 \mu \mathrm{g})$ to the cuvette and used program T-16 of the Nucleofector device to transfect the cells. After transfection, we resuspended cells in serum-free medium and plated them in a 96-well plate. Using a fluorescence-activated cell sorter, we determined transfection efficiency to be $92 \% \pm 4 \%$ and $88 \% \pm 8 \%$ after $24 \mathrm{~h}$ and $48 \mathrm{~h}$, respectively. We determined cell viability $3 \mathrm{~h}, 24 \mathrm{~h}$ and $48 \mathrm{~h}$ after transfection using the CellTiter 96 MTS assay (Promega). The MTS tetrazolium compound is converted by NADPH or NADH produced by metabolically active cells into a colored formazan product and absorbance is measured at $490 \mathrm{~nm}$ using a 96-well-plate reader.

We cultured human adrenal carcinoma SW13.cl.2Vim ${ }^{-}$cells in Dulbecco's modified Eagle medium and Ham's F12 medium (Invitrogen) supplemented with $5 \%$ fetal bovine serum. Transient transfection experiments were done with Lipofectamine as described in the manufacturer's protocol (Invitrogen). We followed previously described immunofluorescence staining procedures ${ }^{22}$ and documented the results with a Nikon Eclipse 800 immunofluorescence microscope and a Spot digital camera. We used mouse monoclonal NR4 antibody to NF-L (Sigma) and rabbit polyclonal antibody to human Hsp27 (Stressgen) for the immunofluorescence studies.

Western blotting. We lysed transfected N2a cells in RIPA buffer $(150 \mathrm{mM}$ $\mathrm{NaCl}, 1 \%$ Nonidet $\mathrm{P}-40,0.5 \%$ sodium deoxycholate, $0.1 \%$ sodium dodecyl sulfate, $50 \mathrm{mM}$ Tris, $\mathrm{pH}$ 8.0) containing protease inhibitors (Roche Diagnostics). Protein concentrations were determined using the micro BCA protein reaction kit (Pierce). We separated proteins, solubilized in SDS-containing sample buffer, by SDS-PAGE in a vertical electrophoresis system (Amersham Biosciences) using a $12 \%$ polyacrylamide gel. After electrophoresis, we transferred proteins to an Immobilon-P membrane (Millipore), washed the membranes in methanol and blocked them for $1 \mathrm{~h}$ at room temperature in 5\% bovine serum albumin (Serva). We then incubated the blots overnight with antibody to HSP27 (M20; Santa Cruz) diluted 1:250 in TBS-Tween and incubated them for $1 \mathrm{~h}$ with antibody to $\operatorname{IgG}$ conjugated to alkaline phosphatase (1:2,500; Sigma). We scanned blots on a STORM 840 scanner (Molecular Dynamics). After stripping the blots, we stained them with antibody to $\beta$-actin (1:10,000; Sigma).

URLs. Online Mendelian Inheritance in Man (OMIM) is available at http://www.ncbi.nlm.nih.gov/entrez/query.fcgi?db=OMIM\&tool=toolbar. To find known genes, expressed-sequence tags and putative new genes, we used National Center for Biotechnology Information Entrez Genome Map Viewer (http://www.ncbi.nlm.nih.gov/), Ensembl Human Genome Server (http:// www.ensembl.org/) and GenBank (http://www.ncbi.nlm.nih.gov/entrez/). We retrieved exon-intron boundaries of the candidate genes from University of California Santa Cruz (http://genome.ucsc.edu). ClustalW multiple protein alignment is available at http://npsa-pbil.ibcp.fr/cgi-bin/npsa_automat.pl?page =npsa_clustalw.html.

Accession numbers. Protein sequences: human HSP27, NP_001531; mouse Hsp27, NP_038588; rat Hsp27, NP_114176; Canis familiaris, P42929; Cricetulus longicaudatus, CAA36036; Gallus gallus, A49181; Poeciliopsis lucida, O13224. The genomic sequences NT_007933, NT_007758 and NT_079593 include the genes HIP1 (NM_005338), AIP1 (NM_012301), CYLN2 (NM_003388 and NM_032421), PTPN12 (NM_002835) and HSPB1 (NM_001540). Genomic sequence NT_023666 contains NEFL (NM_006158). UniGene database: HSBP1, Hs.76067.

Note: Supplementary information is available on the Nature Genetics website.

\section{ACKNOWLEDGMENTS}

We thank the affected individuals and their relatives for participating in this research project; A. Jacobs, E. De Vriendt, V. Van Gerwen, D. Kiraly and M. Jug for technical assistance; and A. Stavljenic-Rukavina for referring one of the families and for institutional support to Z.M.. This research project was supported in part by the Association Française contre les Myopathies, the Association Belge contre les Maladies Neuromusculaires, the Muscular Dystrophy Association, the US National Institutes of Health, Columbia University, the Concerted Research Actions of the Universities of Ghent, Leuven and Antwerp, the Fund for Scientific ResearchFlanders, the Medical Foundation Queen Elisabeth, the Belgian Federal Science Policy Office, the Austrian Science Fund and the Styrian government. I.D. and N.V. are $\mathrm{PhD}$ students supported by the Institute for Science and Technology, Belgium.

\section{COMPETING INTERESTS STATEMENT}

The authors declare that they have no competing financial interests. 
Received 19 February; accepted 12 April 2004

Published online at http://www.nature.com/naturegenetics/

1. Suter, U. \& Scherer, S.S. Disease mechanisms in inherited neuropathies. Nat. Rev Neurosci. 4, 714-726 (2003).

2. Ismailov, S.M. et al. A new locus for autosomal dominant Charcot-Marie-Tooth disease type 2 (CMT2F) maps to chromosome 7q11-q21. Eur. J. Hum. Genet. 9, 646-650 (2001).

3. Zhao, C. et al. Charcot-Marie-Tooth disease type 2A caused by mutation in a microtubule motor KIF1Bbeta. Cell 105, 587-597 (2001).

4. Verhoeven, K. et al. Mutations in the small GTP-ase late endosomal protein RAB7 cause Charcot-Marie-Tooth type 2B neuropathy. Am. J. Hum. Genet. 72, 722-727 (2003).

5. Mersiyanova, I.V. et al. A new variant of Charcot-Marie-Tooth disease type 2 (CMT2E) is probably the result of a mutation in the neurofilament light gene. Am. J. Hum. Genet. 67, 37-46 (2000).

6. Antonellis, A. et al. Glycyl tRNA synthetase mutations in Charcot-Marie-Tooth disease type 2D and distal spinal muscular atrophy type V. Am. J. Hum. Genet. 72 1293-1299 (2003).

7. Klein, C.J. et al. The gene for HMSN2C maps to 12q23-24: a region of neuromuscular disorders. Neurology 60, 1151-1156 (2003).

8. Tang, B. et al. A new locus for autosomal dominant Charcot-Marie-Tooth disease type 2 (CMT2L) maps to chromosome 12q24. Hum. Genet. 114, 527-533 (2004).

9. Nelis, E. et al. Autosomal dominant axonal Charcot-Marie-Tooth disease type 2 (CMT2G) maps to chromosome 12q12-q13.3. J. Med. Genet. 41, 193-197 (2004)

10. Züchner, S. et al. Mutations in the mitochondrial GTPase mitofusin 2 cause CharcotMarie-Tooth neuropathy 2A. Nat. Genet. 36, 449-451 (2004).

11. Sun, X. et al. Interaction of human HSP22 (HSPB8) with other small heat shock proteins. J. Biol. Chem. 279, 2394-2402 (2004)

12. Irobi, J. et al. Hot spot residue in small heat shock protein 22 causes distal motor neu ropathy. Nat. Genet. advance online publication, 2 May 2004 (doi:10.1038/ng1328).

13. Harding, A.E. \& Thomas, P.K. Hereditary distal spinal muscular atrophy. A report on 34 cases and a review of the literature. J. Neurol. Sci. 45, 337-348 (1980).

14. Harding, A.E. \& Thomas, P.K. The clinical features of hereditary motor and sensory neuropathy types I and II. Brain 103, 259-280 (1980).

15. Fontaine, J.M., Rest, J.S., Welsh, M.J. \& Benndorf, R. The sperm outer dense fiber protein is the 10th member of the superfamily of mammalian small stress proteins.
Cell Stress Chaperones 8, 62-69 (2003).

16. Zobel, A.T.C. et al. Distinct chaperone mechanisms can delay the formation of aggresomes by the myopathy-causing R120G alpha B-crystallin mutant. Hum. Mol. Genet. 12, 1609-1620 (2003).

17. Litt, M. et al. Autosomal dominant congenital cataract associated with a missense mutation in the human alpha crystallin gene CRYAA. Hum. Mol. Genet. 7, 471-474 (1998).

18. Bera, S., Thampi, P., Cho, W.J. \& Abraham, E.C. A positive charge preservation at position 116 of alpha A-crystallin is critical for its structural and functional integrity. Biochemistry 41, 12421-12426 (2002).

19. Bruey, J.M. et al. Hsp27 negatively regulates cell death by interacting with cytochrome c. Nat. Cell Biol. 2, 645-652 (2000).

20. Vicart, P. et al. A missense mutation in the alphaB-crystallin chaperone gene causes a desmin-related myopathy. Nat. Genet. 20, 92-95 (1998).

21. Selcen, D. \& Engel, A.G. Myofibrillar myopathy caused by novel dominant negative alpha B-crystallin mutations. Ann. Neurol. 54, 804-810 (2003).

22. Perez-Olle, R., Leung, C.L. \& Liem, R.K.H. Effects of Charcot-Marie-Tooth-linked mutations of the neurofilament light subunit on intermediate filament formation. $J$. Cell Sci. 115, 4937-4946 (2002).

23. Kamradt, M.C., Chen, F. Sam, S. \& Cryns, V.L. The small heat shock protein alpha Bcrystallin negatively regulates apoptosis during myogenic differentiation by inhibiting caspase-3 activation. J. Biol. Chem. 277, 38731-38736 (2002).

24. Benn, S.C. et al. Hsp27 upregulation and phosphorylation is required for injured sensory and motor neuron survival. Neuron 36, 45-56 (2002).

25. Batulan, Z. et al. High threshold for induction of the stress response in motor neurons is associated with failure to activate HSF1. J. Neurosci. 23, 5789-5798 (2003).

26. Vleminckx, V. et al. Upregulation of HSP27 in a transgenic model of ALS. J. Neuropathol. Exp. Neurol. 61, 968-974 (2002).

27. Wang, J. et al. Copper-binding-site-null SOD1 causes ALS in transgenic mice: aggregates of non-native SOD1 delineate a common feature. Hum. Mol. Genet. 12 2753-2764 (2003).

28. Wyttenbach, A. et al. Heat shock protein 27 prevents cellular polyglutamine toxicity and suppresses the increase of reactive oxygen species caused by huntingtin. Hum. Mol. Genet. 11, 1137-1151 (2002).

29. Perng, M.D. et al. Intermediate filament interactions can be altered by HSP27 and alpha B-crystallin. J. Cell Sci. 112, 2099-2112 (1999).

30. Carper, S.W., Rocheleau, T.A., \& Storm, F.K. cDNA sequence of a human heat-shock protein Hsp27. Nucleic Acids Res. 18, 6457 (1990). 